\title{
PFDA-FMEA-VSM application for outsourcing risk analysis
}

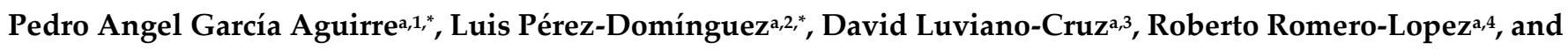 \\ Ernesto Leon-Castrob, ${ }^{\mathrm{b}}$
}

a Departamento de ingeniería Industrial y Manufactura, Universidad Autónoma de Ciudad Juárez, Ciudad
Juárez, Chihuahua, México
b Facultad de Ciencias Económico Administrativas, Universidad Católica de la Santísima Concepción, Concepción, Chile

1 Pedro Angel García Aguirre peagarci@gmail.com ORCID: 0000-0002-4922-0560

2 Dr. Luis Pérez-Domínguez luis.dominguez@uacj.mx ORCID: 0000-0003-2541-4595

3 Dr. David Luviano-Cruz david.luviano@uacj.mx ORCID: 0000-0002-4778-8873

4 Dr. Roberto Romero-López rromero@uacj.mx ORCID: 0000-0003-0859-327X

5 Dr. Ernesto Leon-Castro eleon@ucsc.cl ORCID: 0000-0002-0087-2226

\begin{abstract}
Manufacturing corporations has the acceptance of the Outsourcing Process (OP) to improve industrial activities as well as to archive the revenue objectives, and with this, Risk Analysis (RA) tools are constantly used to assure expected results. Failure Mode and Effect Analysis (FMEA) is one of preferred RA tools, moreover, it is proven that FMEA adds uncertainty because of the human participation at the RA, afterward it is demonstrated that Pythagorean Fuzzy Dimensional Analysis - FMEA - Value Stream Mapping (PFDA-FMEA-VSM) method removes the uncertainty in RA, likewise it aids to the stakeholders for decision making, giving more advantages improving the use of the resources on the project. This document exhibits a real case scenario in a manufacturing firm applying PFDA-FMEA-VSM method adapted for manufacturing OP. The application of PFDA-FMEA-VSM shows solid RA results, removing the human intervention uncertainty added to the risk ranking, gives advantages to the stakeholders for visualize the main risks in detailed diagram, as well as make easier to take better decisions on where to apply resources and mitigate risks during OP.
\end{abstract}

Keywords: Failure Mode and Effect Analysis (FMEA); Pythagorean Fuzzy Dimensional Analysis (PFDA); Value Stream Mapping (VSM); Outsourcing Process (OP); Risk Analysis (RA).

\section{Introduction}

The Outsourcing Process (OP) is nowadays used by the industrial corporations with the intention to simplify their internal manufacturing activities, and to get the expected revenue while a third party manufacture their products [1]. Risk for customers and new products are also important aspects to consider during the OP process [2], likewise, the recent global COVID-19 situation is driving the firms to use OP [3], in addition, global companies look for OP as a business model [4]. Though, OP represents several risks because the intervention of different processes and cross-functional areas are interconnected [5], adding new risks for the firms to manage, making them to look for new Risk Analysis (RA) tools to mitigate the risks and archive the projected goals.

Failure Mode and Effect Analysis (FMEA) is an effective tool to manage risks because it helps to perform an extensive RA, giving a quick guide on what are main risks and how to mitigate them. FMEA is as well a common tool use during the OP [6], however, FMEA is adding uncertainty to the RA since the intervention of the human final decision on what are the risks [7], [8]. Additionally, different studies are showing how to remove the uncertainty while using the FMEA combining Multicriteria methods [9], [10], [11], [12], [13]. Furthermore, Pythagorean Fuzzy Dimensional Analysis - FMEA- Value Stream Mapping 
(PFDA-FMEA-VSM) [14], is a proved method to remove the uncertainty during the RA. PFDA-FMEA-VSM. Furthermore, it adds the advantage to allow the stakeholders taking better decisions before the $\mathrm{OP}$ execution, meanwhile improving where to allocate the budget using just the required resources at the right process step, and risk identified.

This document presents a real case scenario in a manufacturing company, using the PFDA-FMEA-VSM method to perform a RA before manufacturing OP. Furthermore, a comparison between conventional FMEA and PFDA-FMEA-VSM analyzing the differences.

The rest of this document is organized as follows. Section 2, described to the basic concepts required to apply PFDA-FMEA-VSM method. Then section 3, contains the real case scenario, applying PFDA-FMEA-VSM method in a manufacturing company for an OP. Later section 4 covers the results and discussion. Finally, section 5 includes the conclusion of this work.

\section{Basic Concepts}

This segment shows the main concepts used to deploy PFDA-FMEA-VSM method and to apply it in a manufacturing OP. The full details of the used methodology can be found in [14].

Definition 1. Subject Matter Expert team (SMEt), is the group of OP experts which analyze the OP steps and performs the RA. The SMEt should be created by the project leader considering at least three experts, desirable from different functional areas.

Definition 2. SME weights (SMEw) assignation, refers to a value assigned to each SME member, based on their OP experience. The sum of the SME weights must be equal to 1 . It is suggested that the project leader assigned the SME weights.

During this exercise, the SMEt is formed by three senior managers from different functional areas, since the three experts have similar experience in OP, the SMEw are divided by equally.

PFDA-FMEA-VSM method is originally suggested for new product development process risk management, then to allow the adjustment of this methodology to a manufacturing $\mathrm{OP}$, it is required to use next analogies.

1. Phase 1 represents the period between planning stage to the kickoff meeting

2. Phase 2 is the initial segment of the project

3. Phase 3 symbolizes the project implementation

\section{PFDA-FMEA-VSM Application}

This section shows the steps to complete the PFDA-FMEA-VSM method application for an OP in a manufacturing company. Figure 1 depicts the main steps followed to apply PFDA-FMEA-VSM methodology.

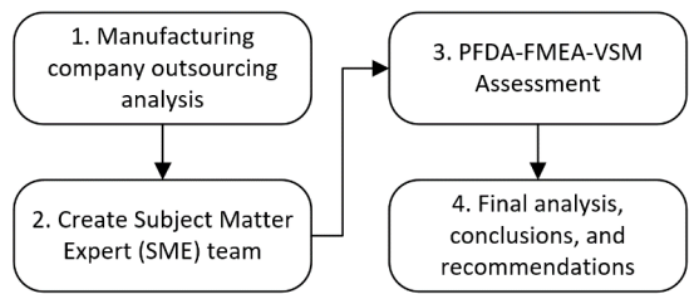

Figure 1. PFDA-FMEA-VSM application summary diagram. 


\section{Results and Discussion}

This section shows the PFDA-FMEA-VSM application result, along with the comparison between the conventional FMEA analysis and the PFDA-FMEA-VSM analysis, this comparison is just for reference and to remark the advantages of the PFDA-FMEA-VSM method. Table 1 shows the list of the risks identified during the SMEt assessment.

Table 1. Potential failure modes (risks) identified by the SMEt.

\begin{tabular}{|c|c|}
\hline ID & Risk \\
\hline 1 & Schedule Execution issues \\
\hline 2 & Continues improvement vs targets \\
\hline 3 & People turnover \\
\hline 4 & Product validation \\
\hline 5 & Supplier capacity issues \\
\hline 6 & Sales projection inaccurate \\
\hline 7 & Product changes not communicated \\
\hline 8 & Poor supplier quality \\
\hline 9 & Manufacturing issues not communicated \\
\hline 10 & Manufacturing errors, bad execution \\
\hline 11 & Poor engineering changes implementation \\
\hline 12 & Project scope changes \\
\hline 13 & KPI bad results from supplier \\
\hline 14 & Lack of right resources assigned \\
\hline 15 & Lack of product history tracking at supplier \\
\hline 16 & Product specs not shared \\
\hline 17 & Slow response to peak of demand \\
\hline 18 & Raw material Long Leadtime \\
\hline 19 & Raw material Long Leadtime \\
\hline 20 & Lack of administrative resources \\
\hline 21 & Slow hiring process \\
\hline 22 & People not hired on time \\
\hline 23 & Lack of right equipment \\
\hline 24 & People turnover during transition \\
\hline 25 & Poor process documentation \\
\hline 26 & Production forecast not well communicated \\
\hline 27 & Product quality does not meet prior transition \\
\hline 28 & Poor raw material management \\
\hline 29 & External agencies approvals long Leadtime \\
\hline 30 & Poor communication Customer-supplier \\
\hline 31 & Missing information during transition \\
\hline 32 & Poor training on new processes \\
\hline 33 & Poor transition product information \\
\hline 34 & Poor knowledge transfer \\
\hline 35 & Administrative resources not properly assigned \\
\hline 36 & Confidential information in risk \\
\hline 37 & Raw material obsolescence not identified \\
\hline 38 & Single manufacturing source \\
\hline 39 & Lack of manufacturing space because of budget \\
\hline 40 & Process capability issues \\
\hline 41 & Schedule execution issues \\
\hline 42 & Poor infrastructure at supplier \\
\hline 43 & Poor engineering changes implementation \\
\hline 44 & People not hired on time \\
\hline 45 & Supplier decommit \\
\hline 46 & Supplier lack of capacity \\
\hline 47 & Logistics issues \\
\hline 48 & Manufacturing certifications issues \\
\hline 49 & ERP system issues \\
\hline 50 & Lack of expertise on manufacturing services \\
\hline 51 & Financial issues (supplier) \\
\hline
\end{tabular}


52 Lack of administrative resources

53 Project transition delay

Figure 2 illustrates the VSM current state.

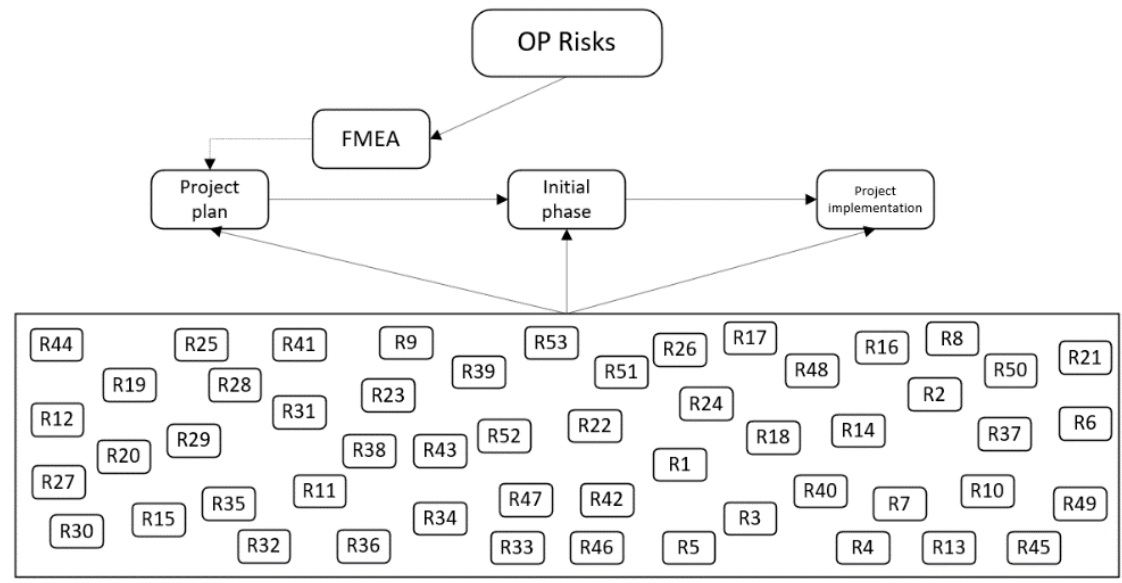

Figure 2. OP VSM current scenario.

Following, the PFDA-FMEA-VSM ranking in Table 2.

Table 2. PFDA-FMEA-VSM ranking results

\begin{tabular}{|c|c|c|}
\hline ID & Risk & $\begin{array}{l}\text { PFDA-FMEA- } \\
\text { VSM Ranking }\end{array}$ \\
\hline 1 & Schedule Execution issues & 17 \\
\hline 2 & Continues improvement vs targets & 13 \\
\hline 3 & People turnover & 37 \\
\hline 4 & Product validation & 35 \\
\hline 5 & Supplier capacity issues & 25 \\
\hline 6 & Sales projection inaccurate & 40 \\
\hline 7 & Product changes not communicated & 42 \\
\hline 8 & Poor supplier quality & 48 \\
\hline 9 & Manufacturing issues not communicated & 25 \\
\hline 10 & Manufacturing errors, bad execution & 8 \\
\hline 11 & Poor engineering changes implementation & 18 \\
\hline 12 & Project scope changes & 46 \\
\hline 13 & KPI bad results from supplier & 47 \\
\hline 14 & Lack of right resources assigned & 5 \\
\hline 15 & Lack of product history tracking at supplier & 10 \\
\hline 16 & Product specs not shared & 24 \\
\hline 17 & Slow response to peak of demand & 52 \\
\hline 18 & Raw material Long Leadtime & 50 \\
\hline 19 & Raw material Long Leadtime & 49 \\
\hline 20 & Lack of administrative resources & 3 \\
\hline 21 & Slow hiring process & 3 \\
\hline 22 & People not hired on time & 16 \\
\hline 23 & Lack of right equipment & 6 \\
\hline 24 & People turnover during transition & 31 \\
\hline 25 & Poor process documentation & 7 \\
\hline 26 & Production forecast not well communicated & 27 \\
\hline 27 & Product quality does not meet prior transition & 8 \\
\hline 28 & Poor raw material management & 36 \\
\hline 29 & External agencies approvals long Leadtime & 12 \\
\hline 30 & Poor communication Customer-supplier & 1 \\
\hline 31 & Missing information during transition & 11 \\
\hline 32 & Poor training on new processes & 38 \\
\hline 33 & Poor transition product information & 30 \\
\hline
\end{tabular}




\begin{tabular}{llc}
\hline 34 & Poor knowledge transfer & 15 \\
35 & Administrative resources not properly assigned & 23 \\
36 & Confidential information in risk & 1 \\
37 & Raw material obsolescence not identified & 33 \\
38 & Single manufacturing source & 41 \\
39 & Lack of manufacturing space because of budget & 18 \\
40 & Process capability issues & 45 \\
41 & Schedule execution issues & 42 \\
42 & Poor infrastructure at supplier & 22 \\
43 & Poor engineering changes implementation & 34 \\
44 & People not hired on time & 32 \\
45 & Supplier decommit & 51 \\
46 & Supplier lack of capacity & 21 \\
47 & Logistics issues & 28 \\
48 & Manufacturing certifications issues & 20 \\
49 & ERP system issues & 39 \\
50 & Lack of expertise on manufacturing services & 14 \\
51 & Financial issues (supplier) & 29 \\
52 & Lack of administrative resources & 44 \\
53 & Project transition delay & 53 \\
\hline
\end{tabular}

Following, the VSM future state is executed. SMEt agreed to select the top 15 risks as potential threads, highlighted in Figure 3.

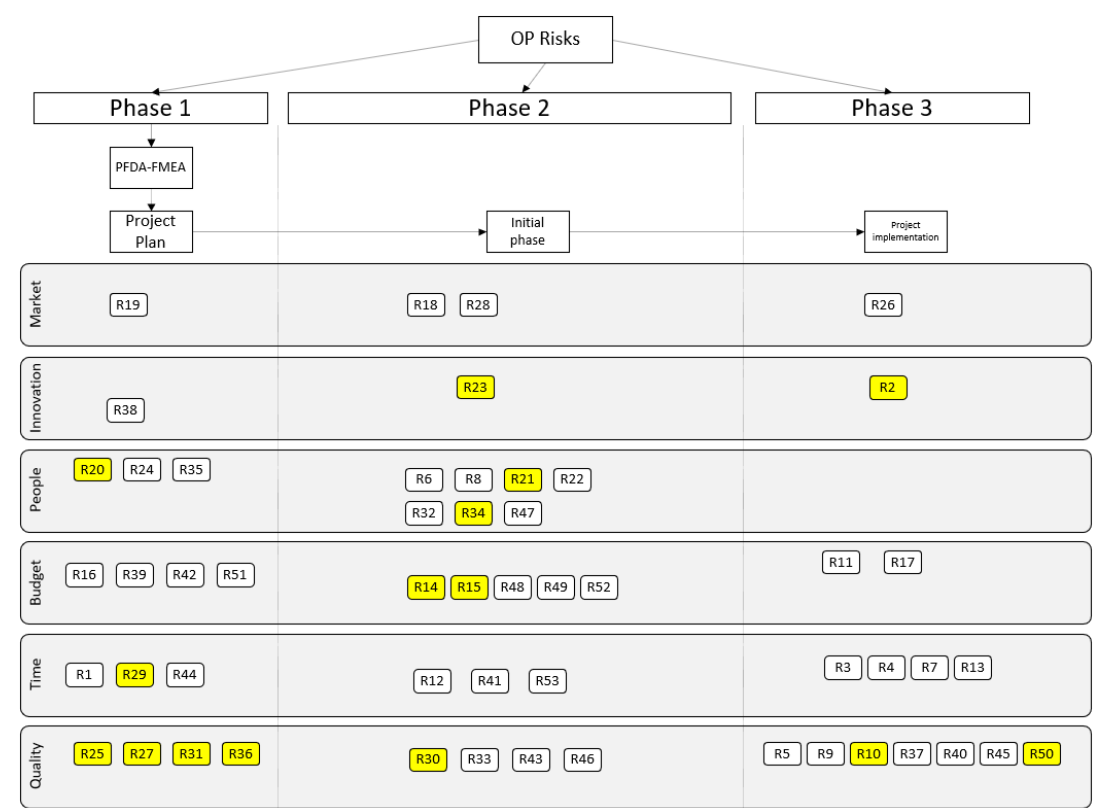

Figure 3. PFDA-FMEA-VSM future state chart result.

For comparison proposes, a conventional FMEA was performed by the same SMEt, Table 3 depicts the FMEA results. SMEt considered to mitigate any risk above RPN of 25.

Table 3. Conventional FMEA assessment by SME.

\begin{tabular}{clcccc}
\hline ID & \multicolumn{1}{c}{ Risk } & S & O & D & RPN \\
\hline 1 & Schedule Execution issues & 5 & 5 & 3 & 75 \\
2 & Continues improvement vs targets & 4 & 2 & 4 & 32 \\
3 & People turnover & 5 & 3 & 5 & 75 \\
4 & Product validation & 5 & 5 & 3 & 75 \\
5 & Supplier capacity issues & 5 & 5 & 3 & 75 \\
6 & Sales projection inaccurate & 5 & 5 & 3 & 75 \\
7 & Product changes not communicated & 5 & 3 & 3 & 45 \\
8 & Poor supplier quality & 5 & 3 & 3 & 45 \\
\hline
\end{tabular}




\begin{tabular}{|c|c|c|c|c|c|}
\hline 9 & Manufacturing issues not communicated & 3 & 2 & 3 & 18 \\
\hline 10 & Manufacturing errors, bad execution & 3 & 2 & 3 & 18 \\
\hline 11 & Poor engineering changes implementation & 3 & 3 & 3 & 27 \\
\hline 12 & Project scope changes & 3 & 3 & 3 & 27 \\
\hline 13 & KPI bad results from supplier & 5 & 5 & 1 & 25 \\
\hline 14 & Lack of right resources assigned & 5 & 5 & 2 & 50 \\
\hline 15 & Lack of product history tracking at supplier & 5 & 5 & 1 & 25 \\
\hline 16 & Product specs not shared & 5 & 5 & 1 & 25 \\
\hline 17 & Slow response to peak of demand & 5 & 5 & 1 & 25 \\
\hline 18 & Raw material Long Leadtime & 5 & 5 & 1 & 25 \\
\hline 19 & Raw material Long Leadtime & 5 & 5 & 1 & 25 \\
\hline 20 & Lack of administrative resources & 5 & 5 & 1 & 25 \\
\hline 21 & Slow hiring process & 5 & 5 & 1 & 25 \\
\hline 22 & People not hired on time & 1 & 2 & 3 & 6 \\
\hline 23 & Lack of right equipment & 1 & 2 & 3 & 6 \\
\hline 24 & People turnover during transition & 1 & 2 & 3 & 6 \\
\hline 25 & Poor process documentation & 5 & 3 & 1 & 15 \\
\hline 26 & Production forecast not well communicated & 5 & 2 & 3 & 30 \\
\hline 27 & Product quality does not meet prior transition & 1 & 5 & 3 & 15 \\
\hline 28 & Poor raw material management & 3 & 1 & 5 & 15 \\
\hline 29 & External agencies approvals long Leadtime & 1 & 1 & 1 & 1 \\
\hline 30 & Poor communication Customer-supplier & 5 & 1 & 3 & 15 \\
\hline 31 & Missing information during transition & 5 & 3 & 1 & 15 \\
\hline 32 & Poor training on new processes & 5 & 3 & 1 & 15 \\
\hline 33 & Poor transition product information & 3 & 5 & 1 & 15 \\
\hline 34 & Poor knowledge transfer & 5 & 3 & 1 & 15 \\
\hline 35 & Administrative resources not properly assigned & 5 & 3 & 1 & 15 \\
\hline 36 & Confidential information in risk & 5 & 3 & 1 & 15 \\
\hline 37 & Raw material obsolescence not identified & 1 & 3 & 3 & 9 \\
\hline 38 & Single manufacturing source & 3 & 3 & 1 & 9 \\
\hline 39 & Lack of manufacturing space because of budget & 1 & 3 & 3 & 9 \\
\hline 40 & Process capability issues & 4 & 2 & 4 & 32 \\
\hline 41 & Schedule execution issues & 4 & 2 & 4 & 32 \\
\hline 42 & Poor infrastructure at supplier & 5 & 1 & 1 & 5 \\
\hline 43 & Poor engineering changes implementation & 3 & 1 & 1 & 3 \\
\hline 44 & People not hired on time & 1 & 3 & 1 & 3 \\
\hline 45 & Supplier decommit & 5 & 3 & 2 & 30 \\
\hline 46 & Supplier lack of capacity & 5 & 3 & 2 & 30 \\
\hline 47 & Logistics issues & 5 & 3 & 2 & 30 \\
\hline 48 & Manufacturing certifications issues & 5 & 3 & 2 & 30 \\
\hline 49 & ERP system issues & 5 & 3 & 2 & 30 \\
\hline 50 & Lack of expertise on manufacturing services & 5 & 3 & 2 & 30 \\
\hline 51 & Financial issues (supplier) & 5 & 3 & 2 & 30 \\
\hline 52 & Lack of administrative resources & 5 & 3 & 2 & 30 \\
\hline 53 & Project transition delay & 5 & 3 & 2 & 30 \\
\hline
\end{tabular}

Conventional FMEA assessment shows 31 identified risks above 25 RPN value, while PFDA-FMEA-VSM top 15 ranking captured the main risks to consider as potential real threats for the OP project.

Using conventional FMEA method, all risks with RPN above 25 should have a mitigation recommended activity, besides, PFDA-FMEA-VSM top 15 optimize the resources at mitigation process, just applying preventive methods where and when required. Table 4 reveals the comparison between PFDA-FMEA-VSM and FMEA.

Table 4. FMEA vs PFDA-FMEA-VSM rankings comparison.

\begin{tabular}{ccccccc}
\hline ID & Risk & S & O & D & RPN & $\begin{array}{c}\text { PFDA-FMEA- } \\
\text { VSM Ranking }\end{array}$ \\
\hline 1 & Schedule Execution issues & 5 & 5 & 3 & 75 & 17 \\
\hline
\end{tabular}




\begin{tabular}{|c|c|c|c|c|c|c|}
\hline 2 & Continues improvement vs targets & 4 & 2 & 4 & 32 & 13 \\
\hline 3 & People turnover & 5 & 3 & 5 & 75 & 37 \\
\hline 4 & Product validation & 5 & 5 & 3 & 75 & 35 \\
\hline 5 & Supplier capacity issues & 5 & 5 & 3 & 75 & 25 \\
\hline 6 & Sales projection inaccurate & 5 & 5 & 3 & 75 & 40 \\
\hline 7 & Product changes not communicated & 5 & 3 & 3 & 45 & 42 \\
\hline 8 & Poor supplier quality & 5 & 3 & 3 & 45 & 48 \\
\hline 9 & Manufacturing issues not communicated & 3 & 2 & 3 & 18 & 25 \\
\hline 10 & Manufacturing errors, bad execution & 3 & 2 & 3 & 18 & 8 \\
\hline 11 & Poor engineering changes implementation & 3 & 3 & 3 & 27 & 18 \\
\hline 12 & Project scope changes & 3 & 3 & 3 & 27 & 46 \\
\hline 13 & KPI bad results from supplier & 5 & 5 & 1 & 25 & 47 \\
\hline 14 & Lack of right resources assigned & 5 & 5 & 2 & 50 & 5 \\
\hline 15 & Lack of product history tracking at supplier & 5 & 5 & 1 & 25 & 10 \\
\hline 16 & Product specs not shared & 5 & 5 & 1 & 25 & 24 \\
\hline 17 & Slow response to peak of demand & 5 & 5 & 1 & 25 & 52 \\
\hline 18 & Raw material Long Leadtime & 5 & 5 & 1 & 25 & 50 \\
\hline 19 & Raw material Long Leadtime & 5 & 5 & 1 & 25 & 49 \\
\hline 20 & Lack of administrative resources & 5 & 5 & 1 & 25 & 3 \\
\hline 21 & Slow hiring process & 5 & 5 & 1 & 25 & 3 \\
\hline 22 & People not hired on time & 1 & 2 & 3 & 6 & 16 \\
\hline 23 & Lack of right equipment & 1 & 2 & 3 & 6 & 6 \\
\hline 24 & People turnover during transition & 1 & 2 & 3 & 6 & 31 \\
\hline 25 & Poor process documentation & 5 & 3 & 1 & 15 & 7 \\
\hline 26 & Production forecast not well communicated & 5 & 2 & 3 & 30 & 27 \\
\hline 27 & Product quality not meet prior transition & 1 & 5 & 3 & 15 & 8 \\
\hline 28 & Poor raw material management & 3 & 1 & 5 & 15 & 36 \\
\hline 29 & External agencies approvals long Leadtime & 1 & 1 & 1 & 1 & 12 \\
\hline 30 & Poor communication Customer-supplier & 5 & 1 & 3 & 15 & 1 \\
\hline 31 & Missing information during transition & 5 & 3 & 1 & 15 & 11 \\
\hline 32 & Poor training on new processes & 5 & 3 & 1 & 15 & 38 \\
\hline 33 & Poor transition product information & 3 & 5 & 1 & 15 & 30 \\
\hline 34 & Poor knowledge transfer & 5 & 3 & 1 & 15 & 15 \\
\hline 35 & Administrative resources not properly assigned & 5 & 3 & 1 & 15 & 23 \\
\hline 36 & Confidential information in risk & 5 & 3 & 1 & 15 & 1 \\
\hline 37 & Raw material obsolescence not identified & 1 & 3 & 3 & 9 & 33 \\
\hline 38 & Single manufacturing source & 3 & 3 & 1 & 9 & 41 \\
\hline 39 & Lack of manufacturing space because of budget & 1 & 3 & 3 & 9 & 18 \\
\hline 40 & Process capability issues & 4 & 2 & 4 & 32 & 45 \\
\hline 41 & Schedule execution issues & 4 & 2 & 4 & 32 & 42 \\
\hline 42 & Poor infrastructure at supplier & 5 & 1 & 1 & 5 & 22 \\
\hline 43 & Poor engineering changes implementation & 3 & 1 & 1 & 3 & 34 \\
\hline 44 & People not hired on time & 1 & 3 & 1 & 3 & 32 \\
\hline 45 & Supplier decommit & 5 & 3 & 2 & 30 & 51 \\
\hline 46 & Supplier lack of capacity & 5 & 3 & 2 & 30 & 21 \\
\hline 47 & Logistics issues & 5 & 3 & 2 & 30 & 28 \\
\hline 48 & Manufacturing certifications issues & 5 & 3 & 2 & 30 & 20 \\
\hline 49 & ERP system issues & 5 & 3 & 2 & 30 & 39 \\
\hline 50 & Lack of expertise on manufacturing services & 5 & 3 & 2 & 30 & 14 \\
\hline 51 & Financial issues (supplier) & 5 & 3 & 2 & 30 & 29 \\
\hline 52 & Lack of administrative resources & 5 & 3 & 2 & 30 & 44 \\
\hline 53 & Project transition delay & 5 & 3 & 2 & 30 & 53 \\
\hline
\end{tabular}

\section{Conclusions}

A recurrent issue identifying risks in $\mathrm{OP}$, is the uncertainty added by the human intervention ranking the risks, moreover by using PFDA-FMEA-VSM method, this problem is solved and improves OP with significant advantages over the conventional FMEA. Following, a list of the primary benefits of using PFDA-FMEA-VSM for OP. 
- Ranking uncertainty removed

- Clear visibility on the risks to be mitigated

- Optimize resources mitigating just the major risks

- Visual risks identification, where and when is the risk

PFDA-FMEA-VSM method was at first used to new product introduction process, moreover, this application reveals that it is well adapted to OP making clear and easier the OP. Likewise, there is a value added using this method, because of the risk classification by area and the project period.

Furthermore, future works are considered applying and adapting PFDA-FMEAVSM to other processes, as well as trying to automate the process using a programmed software.

Author Contributions: Formal analysis, L.P.-D.; Investigation, L.P.-D.; Methodology, P.A.G.A.; Project administration, R. R.-L.; Resources, R. R.-L.; Supervision, E.L.-C.; Validation, D.L.-C.; Visualization, D.L.-C.; Writing - original draft, P.A.G.A.; Writing - review \& editing, L.P.-D. All authors have read and agreed to the published version of the manuscript.

Funding: This research was funded by CONSEJO NACIONAL DE CIENCIA Y TECNOLOGIA (Conacyt), No. CVU: 576388.

Data Availability Statement: Not apply

Acknowledgments: Not apply

Conflicts of Interest: The authors declare no conflict of interest.

\section{References}

[1] H. Huang, F. Liu, and P. Zhang, "To outsource or not to outsource? Warranty service provision strategies considering competition, costs and reliability," Int. J. Prod. Econ., vol. 242, no. May, p. 108298, 2021, doi: 10.1016/j.ijpe.2021.108298.

[2] V. Good and R. J. Calantone, "When to outsource the sales force for new products," Ind. Mark. Manag., vol. 82, no. February 2018, pp. 106-116, 2019, doi: 10.1016/j.indmarman.2019.02.010.

[3] C.-A. Tsai, T.-H. Ho, J.-S. Lin, C.-C. Tu, and C.-W. Chang, “Model for Evaluating Outsourcing Logistics Companies in the COVID-19 Pandemic," Logistics, vol. 5, no. 3, p. 64, 2021, doi: 10.3390/logistics5030064.

[4] G. Jadraque, “Knowledge Management System as a Business Process Outsourcing Approach," SSRN Electron. J., 2020, doi: $10.2139 /$ ssrn.3608815.

[5] S. Strese, M. W. Meuer, T. C. Flatten, and M. Brettel, “Organizational antecedents of cross-functional coopetition: The impact of leadership and organizational structure on cross-functional coopetition," Ind. Mark. Manag., vol. 53, pp. 42-55, 2016, doi: 10.1016/j.indmarman.2015.11.006.

[6] P. Liu and S. Wang, "Evolutionary Game Analysis of Cold Chain Logistics Outsourcing of Fresh Food Enterprises with Operating Risks," IEEE Access, vol. 8, pp. 127094-127103, 2020, doi: 10.1109/ACCESS.2020.3006730.

[7] Z. Liu and F. Xiao, "An intuitionistic evidential method for weight determination in FMEA based on belief entropy," Entropy, vol. 21, no. 2, 2019, doi: 10.3390/e21020211.

[8] J. Oehmen, A. Guenther, J. W. Herrmann, J. Schulte, and P. Willumsen, "RISK MANAGEMENT in PRODUCT DEVELOPMENT: RISK IDENTIFICATION, ASSESSMENT, and MITIGATION - A LITERATURE REVIEW," Proc. Des. Soc. Des. Conf., vol. 1, no. iii, pp. 657-666, 2020, doi: 10.1017/dsd.2020.27.

[9] S. Almeraz-Durán, L. A. Pérez-Domínguez, D. Luviano-Cruz, J. I. Hernández Hernández, R. Romero López, and D. J. ValleRosales, “A proposed framework for developing fmea method using pythagorean fuzzy codas," Symmetry (Basel)., vol. 13, 
no. 12 , pp. 1-20, 2021, doi: 10.3390/sym13122236.

[10] Y. M. Wang, K. S. Chin, G. K. K. Poon, and J. B. Yang, "Risk evaluation in failure mode and effects analysis using fuzzy weighted geometric mean," Expert Syst. Appl., vol. 36, no. 2 PART 1, pp. 1195-1207, 2009, doi: 10.1016/j.eswa.2007.11.028.

[11] P. Garcia, L. Perez-Dominguez, D. Luviano-cruz, J. Solano, E. Martinez, and M. Callejas-cuervo, "PFDA-FMEA, an Integrated Method Improving FMEA Assessment in Product Design," Appl. Sci. MDPI, vol. 11, no. 1406, 2021.

[12] J. H. Dahooie, A. S. Vanaki, H. R. Firoozfar, E. K. Zavadskas, and A. Čereška, "An extension of the failure mode and effect analysis with hesitant fuzzy sets to assess the occupational hazards in the construction industry," Int. J. Environ. Res. Public Health, vol. 17, no. 4, 2020, doi: 10.3390/ijerph17041442.

[13] M. Akram, A. Luqman, and J. C. R. Alcantud, "Risk evaluation in failure modes and effects analysis: hybrid TOPSIS and ELECTRE I solutions with Pythagorean fuzzy information,” Neural Comput. Appl., vol. 33, no. 11, pp. 5675-5703, 2021, doi: 10.1007/s00521-020-05350-3.

[14] P. A. García Aguirre, L. Pérez-Domínguez, D. Luviano-Cruz, E. M. Gómez, I. J. C. P. Olguin, and J. O. D. Ramírez, “Risk assessment with value added pythagorean fuzzy failure mode and effect analysis for stakeholders," IEEE Access, vol. 9, pp. 149560-149568, 2021, doi: 10.1109/ACCESS.2021.3124480. 